\title{
Genome Analysis of A Novel South African Cydia pomonella granulovirus (CpGV-SA) with Resistance-Breaking Potential
}

\author{
Boitumelo Motsoeneng ${ }^{1, \dagger}{ }^{,}$Michael D. Jukes ${ }^{1,2, *,+} \oplus$, Caroline M. Knox ${ }^{1}$, Martin P. Hill ${ }^{2}$ \\ and Sean D. Moore 2,3 \\ 1 Department of Biochemistry and Microbiology, Rhodes University, P.O. Box 94, \\ Grahamstown 6140, South Africa \\ 2 Centre for Biological Control, Department of Zoology and Entomology, Rhodes University, P.O. Box 94, \\ Grahamstown 6140, South Africa \\ 3 Citrus Research International, P.O. Box 5095, Walmer, Port Elizabeth 6065, South Africa \\ * Correspondence: mrmdjukes@gmail.com \\ + These authors contributed equally to this article.
}

Received: 31 May 2019; Accepted: 24 June 2019; Published: 18 July 2019

\begin{abstract}
The complete genome of an endemic South African Cydia pomonella granulovirus isolate was sequenced and analyzed. Several missing or truncated open reading frames (ORFs) were identified, including a 24 bp deletion in the pe38 gene which is reported to be associated with type I resistance-breaking potential. Comparison of single nucleotide polymorphisms (SNPs) with five other fully sequenced CpGV isolates identified 67 unique events, 47 of which occurred within ORFs, leading to several amino acid changes. Further analysis of single nucleotide variations (SNVs) within CpGV-SA revealed that this isolate consists of mixed genotypes. Phylogenetic analysis using complete genome sequences placed CpGV-SA basal to M, I12 and E2 and distal to S and I07 but with no distinct classification into any of the previously defined CpGV genogroups. These results suggest that CpGV-SA is a novel and genetically distinct isolate with significant potential as a biopesticide for management of codling moth (CM), not only in South Africa, but potentially in other pome fruit producing countries, particularly where $\mathrm{CM}$ resistance to $\mathrm{CpGV}$ has been reported.
\end{abstract}

Keywords: Cydia pomonella granulovirus; codling moth; single nucleotide polymorphism (SNP); single nucleotide variation $(\mathrm{SNV})$

\section{Introduction}

The codling moth, Cydia pomonella (CM; Lepidoptera: Tortricidae), is an economically important agricultural pest causing extensive damage to pome fruit production in most parts of the temperate world, including South Africa [1,2]. Among the options available for management of this pest is the Cydia pomonella granulovirus, $\mathrm{CpGV}$, belonging to the dsDNA family Baculoviridae (genus Betabaculovirus), which is now registered in 34 countries worldwide as a highly selective, effective and environmentally safe biological control agent [3]. Since its discovery in Mexico in 1963 [4], several genetically distinct isolates of CpGV have been described from various regions of the world and have been classified into phylogenetic genome groups A-E. Representative examples of members of different genome groups include M (group A), E2 (group B), I07 (group C), I12 (group D) and S (group E) [5,6]. Currently, there are six full CpGV genome sequences from different geographic origins available on the NCBI database for comparison with novel isolates. Recently, these genomes have been compared to infer intra-species diversity and to create a genome-wide map of CpGV based on specific positions of single nucleotide polymorphisms (SNPs) within each genome [7]. 
Commercial CpGV products have been used since the late 1980s for the control of CM in pome fruit growing areas worldwide and, initially, most of them contained the Mexican isolate, CpGV-M, as the active ingredient [8]. In 2004 and 2005, resistance in CM field populations was reported in France and Germany for the first time $[9,10]$ and subsequently confirmed by laboratory bioassays $[11,12]$. This mode of resistance, termed Type I [13], was found to be isolate-specific and directed only at CpGV-M (genogroup A). Furthermore, sequence analysis confirmed that it was associated with the presence of a 24 bp motif in the pe38 gene, which was missing in so-called "resistance-breaking" isolates that could overcome CM resistance [6,14-16]. The observation of widespread CpGV resistance prompted many investigations across Europe, leading to field trials and alternative CpGV commercial formulations for CM management [3,14-20]. Recently, infection studies and crossing experiments with laboratory CM strains revealed additional modes of resistance, namely, Type II directed against most known CpGV isolates except those belonging to genotype B [13] and a more complex Type III resistance, which appeared to target at least two CpGV genome groups, A and E [21]. These observations highlight the importance of further understanding the genetic basis for resistance and the co-evolution of virus and host if CpGV-based management is to be sustained as one of the most effective control measures in pome fruit production globally.

The South African pome fruit industry is a well-established, multi-billion Rand operation, cultivating apples and pears for both local consumption and export mainly to Europe, the Far East, Asia, and the rest of the African continent [22]. Despite its reputation for producing high quality fruit, the industry is threatened by CM, which was first introduced into South Africa in 1885 and has become established in the country since then [2,23]. Baculovirus products including Madex (Andermatt, Switzerland) and Carpovirusine (Arysta Lifescience), all formulated with CpGV-M, have been registered in South Africa and are used to control CM as part of an integrated pest management programme [24,25]. Alarm over reports of widespread resistance in field populations of CM to CpGV-M in Europe led to bioprospecting for endemic South African CpGV isolates [26]. Here, we report on the genome analysis of a novel CpGV isolate which was recovered from diseased larvae in the Free State Province of South Africa, where no CpGV products were previously applied. The potential of this isolate for management of resistance, should it occur in South Africa following intensive application of CpGV-M based biopesticides in the field, is discussed.

\section{Materials and Methods}

\subsection{Genome Sequencing and Assembly}

Genomic DNA was extracted from purified CpGV-SA occlusion bodies (OBs) recovered from field-collected larvae [26,27]. Approximately $100 \mathrm{ng}$ of CpGV-SA genomic DNA was sequenced using a MiSeq Desktop Sequencer (Illumina, San Diego, CA, USA). Geneious R7 (Biomatters, Auckland, New Zealand) was used to conduct a de novo assembly, which produced 10,000 high quality contigs from 691,903 paired reads with an average coverage of $545.4 \pm 191.3$. The contigs were then mapped to the reference sequence, CpGV-M1, an in vivo cloned genotype of the Mexican isolate [28]. The consensus sequence generated was annotated using both CpGV-M1 and CpGV-M [6]. The assembled sequence of the CpGV-SA genome (NCBI GenBank: MN075941) was validated by comparing in silico and in vitro restriction endonuclease profiles [27]. The CPGV-SA genome was then used to detect single nucleotide variations (SNVs), within the isolate, and to analyse single nucleotide polymorphisms (SNPs) across geographically distinct isolates of CpGV.

\subsection{Comparative Single Nucleotide Polymorphism Analysis}

To identify SNPs (intra-species variation), a nucleotide alignment of CpGV-SA and CpGV-M, -I07, -I12, -S and -E2 complete genome sequences was first generated using ClustalW in Geneious R11. SNPs were identified by comparing each sequence against the others using the Variation/SNP tool in Geneious R11 with the minimum variant frequency set to 0.1 and effects on translations analysed. 
The resulting SNP data were exported to Excel 365 (Microsoft, Redmond, WA, USA) and analysed. SNPs were only considered for nucleotides in which all six sequences successfully aligned and were not disrupted by small insertions or deletions (Indel).

\subsection{Genotypic Variation within the CpGV-SA Isolate}

The above-mentioned raw Illumina data were reanalysed in Geneious R11 with paired reads error corrected and normalized using the BBNorm plugin. Reads were de novo assembled and the top 1000 contigs generated further, dissolved and mapped to the assembled CpGV-SA genome sequence. SNVs (variation within CpGV-SA) were detected amongst the aligned reads using the variation detection tool in Generous R11. A total of 972,303 reads, with Q20, Q30 and Q40 values of 91.0\%, 82.9\% and $39.6 \%$, respectively, successfully mapped to the complete genome sequence. Data generated were exported to Excel 365 and analysed.

\subsection{Phylogenetic Analysis}

A new nucleotide alignment consisting of complete genome sequences from CpGV-SA, -M, -I07, -I12, -S, -E2 and Cryptophlebia leucotreta granulovirus (CrleGV) (NC_005068), as an outlier, was used to construct a minimum evolution (ME) phylogenetic tree. The phylogenetic tree was inferred by using 1000 bootstrap replicates with a close-neighbor-interchange algorithm with an initial tree obtained by neighbor joining. The analysis was conducted using MEGA X (Version 10) software [29].

\section{Results}

\subsection{CpGV-SA Genome Characteristics}

The complete genome sequence for CpGV-SA is 123,595 bp with coding regions of 110,551 bp $(89.45 \%)$. The CPGV-SA genome sequence was initially annotated using the gene data from the reference sequence, CpGV-M1. However, the open reading frames (ORFs) of CpGV-M that were determined as different from CpGV-M1 were chosen as the annotations, due to having higher percentage identities when assessing the nucleotide and amino acid sequences. The genome has a $45.3 \%$ GC content and is comprised of 141 ORFs out of the 142 identified in CpGV-M. A total of 57 ORFs within the CpGV-SA genome are $100 \%$ identical to the corresponding ORFs in the CpGV-M isolate. ORFs cp25 and cp38 of CpGV-M1, encoded less than 50 amino acids (aa) and are therefore regarded as absent from the CpGV-SA genome [28,30].

Further analysis revealed that ORF cp24 (pe38) was truncated with a 24 bp deletion similar to CpGV-E2, -I07, -I12, and -S. Truncations were also observed in ORFs cp18, cp27, cp31, cp37 and cp62 by 112, 267, 91, 266 and 159 aa, respectively, when compared to CpGV-M. The truncation in ORFs 27 and 62 was particularly severe with approximately 60.6 and $83.6 \%$ of the coding region lost. Extended ORFs were identified for ORF cp6 and cp63 by 18 and 25 aa, respectively, in relation to CpGV-M. ORFs cp28/29, 50/51 and 129/130 were fused into single ORFs. Like most CpGV isolates, cp52 was split into two frames, cp52a and cp52b.

Several unique INDELs within ORFs were also identified in the CpGV-SA genome sequence. These include a 3 bp deletion in cp50/cp51 and cp102 at bases 42325 to 42327 and 85423 to 85425 in CpGV-M, respectively, with the former INDEL also present in CpGV-S and I07. Similarly, a deletion of $3 \mathrm{bp}$ in cp115 was identified to disrupt the stop codon, located at positions 98349 to 98351 in CpGV-M, resulting in an extension of the ORF by 9 amino acids.

\subsection{Comparative Single Nucleotide Polymorphism Analysis}

A comparison of CpGV-SA against all other known CpGV isolates with complete genome sequences was conducted to identify novel SNPs and amino acid changes (Table 1). The pairwise identity of CpGV-SA to CpGV- E2, -I07, -I12, -M, and -S was 98.07\%, 96.23\%, 98.26\%, 98.9\% and $98.99 \%$, respectively. 
Table 1. Alignment of 67 novel SNPs identified between CpGV-SA and CpGV-E2, -I07, -I12, -M, and -S, grouped as CpGV. Positions for each nucleotide are given relative to the CpGV-M and CpGV-SA sequences. The corresponding ORF numbers are provided above the alignment for SNPs occurring within coding regions while any resulting amino acid substitutions are shown below. Nucleotides: $\mathrm{A}=$ Adenine, $\mathrm{C}=\mathrm{Cytosine}, \mathrm{G}=\mathrm{Guanine}, \mathrm{T}=\mathrm{Thymine}$; Ambiguous nucleotides: $\mathrm{Y}=\mathrm{C}$ or $\mathrm{T}$ and $\mathrm{M}=\mathrm{A}$ or $\mathrm{C}$ and amino acids: $\mathrm{A}=$ Alanine, $\mathrm{R}=$ Arginine, $\mathrm{N}=$ Asparagine, $\mathrm{Q}=$ Glutamine $, \mathrm{H}=\mathrm{Histidine}, \mathrm{I}=\mathrm{I}$ soleucine $, \mathrm{K}=\mathrm{Lysine}, \mathrm{P}=\mathrm{Proline}, \mathrm{S}=\mathrm{Serine}, \mathrm{T}=$ Threonine, and $\mathrm{V}=$ Valine.

\begin{tabular}{|c|c|c|c|c|c|c|c|c|c|c|c|c|c|c|c|c|c|c|c|c|c|c|c|c|c|c|c|c|c|c|c|c|c|}
\hline $\begin{array}{l}\text { CpGV-M } \\
\text { Position }\end{array}$ & $\begin{array}{l}\stackrel{L}{L} \\
\stackrel{M}{c}\end{array}$ & مै & $\begin{array}{l}1 \\
0 \\
0\end{array}$ & ठ્ठ & 栾 & 各 & 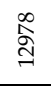 & 产 & 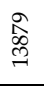 & $\stackrel{\infty}{\stackrel{F}{\sharp}}$ & 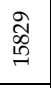 & $\begin{array}{l}\infty \\
\stackrel{0}{\Xi}\end{array}$ & 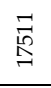 & 离 & ֻิे & 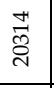 & Iี & 帒 & 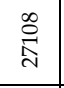 & $\begin{array}{l}\widehat{0} \\
\text { ळ్ల } \\
0\end{array}$ & $\frac{m}{9}$ & 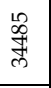 & $\begin{array}{l}\vec{ल} \\
\infty \\
\infty \\
\infty\end{array}$ & 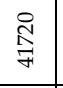 & 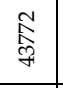 & 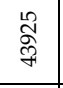 & 孪 & 产 & 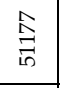 & $\begin{array}{l}\infty \\
\stackrel{\infty}{\mathrm{m}} \\
\mathrm{n}\end{array}$ & $\underset{\text { F }}{\stackrel{9}{*}}$ & 密 & $\begin{array}{l}\text { 范 } \\
\text { in } \\
\text { in }\end{array}$ \\
\hline $\begin{array}{l}\text { CpGV-SA } \\
\text { Position }\end{array}$ & 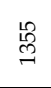 & 点 & 今ે & సू & 究 & $\underset{\infty}{\tilde{I}}$ & $\begin{array}{l}\text { 赵 } \\
\text { c } \\
-1\end{array}$ & 吉 & बे & $\begin{array}{l}\infty \\
\stackrel{8}{\leftrightarrows}\end{array}$ & 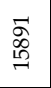 & $\stackrel{\text { ڤે }}{\stackrel{2}{\Xi}}$ & 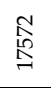 & $\begin{array}{l}\infty \\
\infty \\
\stackrel{\sim}{*}\end{array}$ & ले & $\begin{array}{l}\text { ले } \\
\text { ర్ }\end{array}$ & ले & 市 & 离 & 今 & $\begin{array}{l}\infty \\
\text { o̊ } \\
\text { के }\end{array}$ & 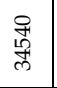 & $\begin{array}{l}\infty \\
\infty \\
\infty \\
\infty \\
\infty\end{array}$ & $\begin{array}{l}\frac{10}{1} \\
\frac{1}{7}\end{array}$ & $\begin{array}{l}\text { aे } \\
\text { o } \\
\text { के }\end{array}$ & $\begin{array}{l}\triangleright \\
\infty \\
\check{\xi}\end{array}$ & $\begin{array}{l}8 \\
\stackrel{b}{*} \\
\text { in }\end{array}$ & $\begin{array}{l}0 \\
\stackrel{0}{7} \\
\text { in }\end{array}$ & $\begin{array}{l}\text { ลิ } \\
\text { กิ }\end{array}$ & $\begin{array}{l}\infty \\
\stackrel{\infty}{a} \\
i n\end{array}$ & $\begin{array}{l}\text { त्रे } \\
\stackrel{\omega}{\omega}\end{array}$ & 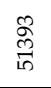 & $\underset{f}{\text { 舟 }}$ \\
\hline ORF & $\infty$ & $\wedge$ & \multicolumn{3}{|c|}{$\stackrel{\circ}{\circ}$} & $=$ & \multicolumn{2}{|c|}{$\triangle$} & & $\stackrel{\infty}{\sim}$ & กิ & \multicolumn{2}{|c|}{$\pi$} & & & & & ल & & & & F & f & \multicolumn{3}{|c|}{ in } & \multicolumn{2}{|c|}{5} & & & & & \\
\hline CpGV-SA & A & $\mathrm{T}$ & A & $\mathrm{T}$ & A & A & $\mathrm{T}$ & A & G & $\mathrm{T}$ & $\mathrm{T}$ & C & A & A & $\mathrm{T}$ & A & A & $\mathrm{T}$ & A & A & A & A & A & $\mathrm{T}$ & $\mathrm{T}$ & A & $\mathrm{T}$ & C & $\mathrm{T}$ & $\mathrm{T}$ & $\mathrm{T}$ & $\mathrm{T}$ & C \\
\hline CpGV & G & A & G & C & G & G & C & C & A & C & C & $\mathrm{T}$ & G & $\mathrm{T}$ & C & G & $\mathrm{T}$ & C & G & G & C & G & $\mathrm{T}$ & C & C & C & A & G & G & C & A & A & $\mathrm{T}$ \\
\hline $\begin{array}{l}\text { Substitution } \\
\text { SA -> Ref }\end{array}$ & & 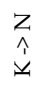 & $\stackrel{\hat{i}}{i}$ & & $\begin{array}{l}\hat{\hat{n}} \\
\hat{n}\end{array}$ & & & & & & $\begin{array}{l}\overleftrightarrow{\hat{i}} \\
\hat{H}\end{array}$ & & $\begin{array}{l}\stackrel{\mathbb{i}}{H} \\
\stackrel{H}{H}\end{array}$ & & & & & $\begin{array}{l}\overleftrightarrow{\hat{\lambda}} \\
\stackrel{>}{>}\end{array}$ & & & & & $\begin{array}{l}Z \\
\hat{i} \\
-\end{array}$ & & & $\begin{array}{l}\vec{T} \\
\hat{a} \\
a\end{array}$ & $\overrightarrow{\hat{z}}$ & $\stackrel{\overrightarrow{\hat{n}}}{\simeq}$ & & & & & \\
\hline
\end{tabular}

\begin{tabular}{|c|c|c|c|c|c|c|c|c|c|c|c|c|c|c|c|c|c|c|c|c|c|c|c|c|c|c|c|c|c|c|c|c|c|c|}
\hline $\begin{array}{l}\text { CpGV-M } \\
\text { Position }\end{array}$ & $\begin{array}{l}\hat{⿵} \\
\hat{n} \\
\text { in }\end{array}$ & $\begin{array}{l}0 \\
\infty \\
\infty \\
\infty \\
n\end{array}$ & 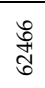 & 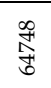 & $\underset{0}{\stackrel{+}{E}}$ & $\begin{array}{l}\text { aे } \\
\text { ò } \\
\text { b. }\end{array}$ & 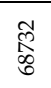 & 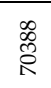 & 免 & đू & $\begin{array}{l}\infty \\
\stackrel{0}{0} \\
\stackrel{1}{1}\end{array}$ & $\begin{array}{l}\hat{\aleph} \\
\stackrel{\infty}{\wedge} \\
\hat{N}\end{array}$ & 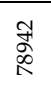 & 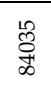 & 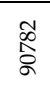 & 吕 & $\frac{H}{\#}$ & 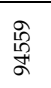 & $\begin{array}{l}\hat{\sigma} \\
\hat{\Omega}\end{array}$ & $\begin{array}{l}\text { के } \\
\text { مू }\end{array}$ & 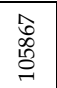 & $\stackrel{m}{\overrightarrow{0}}$ & $\begin{array}{l}\infty \\
0 \\
0 \\
0\end{array}$ & $\begin{array}{l}\text { 蒿 } \\
\stackrel{\sigma}{=}\end{array}$ & 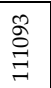 & $\begin{array}{l}\stackrel{0}{1} \\
\exists\end{array}$ & $\begin{array}{l}\stackrel{\mathscr{n}}{\mathscr{n}} \\
\stackrel{0}{\Xi}\end{array}$ & $\begin{array}{l}\text { th } \\
\text { 点 } \\
\stackrel{7}{7}\end{array}$ & $\begin{array}{l}\text { त్ } \\
\stackrel{6}{=}\end{array}$ & 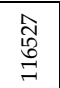 & $\begin{array}{l}\text { 茴 } \\
\text { 亲 }\end{array}$ & 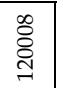 & $\begin{array}{l}\text { ్ㅗㅁ } \\
\text { đั }\end{array}$ & 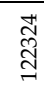 \\
\hline $\begin{array}{l}\text { CpGV-SA } \\
\text { Position }\end{array}$ & 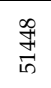 & 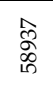 & $\begin{array}{l}\text { ते } \\
\text { त్ర }\end{array}$ & 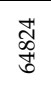 & 各 & م્ & $\begin{array}{l}\infty \\
\infty \\
\infty \\
0 \\
0\end{array}$ & 草 & 喜 & $\begin{array}{l}\infty \\
\text { 定 } \\
\text { N }\end{array}$ & 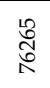 & 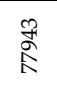 & $\underset{\substack{\infty \\
\vdots}}{\stackrel{2}{人}}$ & $\underset{\infty}{\vec{F}}$ & 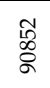 & $\begin{array}{l}\text { สूँ } \\
\text { ूू }\end{array}$ & $\begin{array}{l}\text { 索 } \\
\text { ç }\end{array}$ & $\begin{array}{l}\text { 心్ర } \\
\text { \$ั }\end{array}$ & $\begin{array}{l}\text { J్ } \\
\text { 心ू }\end{array}$ & 兽 & 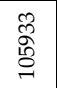 & $\begin{array}{l}\stackrel{0}{0} \\
\stackrel{0}{0}\end{array}$ & 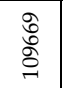 & $\begin{array}{l}\text { : } \\
\stackrel{0}{\circ}\end{array}$ & $\stackrel{\infty}{\stackrel{\infty}{\Xi}}$ & $\begin{array}{l}\stackrel{\mathscr{N}}{\Xi} \\
\stackrel{ल}{\Xi}\end{array}$ & $\begin{array}{l}8 \\
: \\
\exists\end{array}$ & $\begin{array}{l}\infty \\
\stackrel{0}{0} \\
\stackrel{0}{7}\end{array}$ & 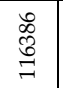 & 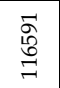 & $\stackrel{ }{\stackrel{ }{\Xi}}$ & $\begin{array}{l}\text { Nิे } \\
\text { స్ }\end{array}$ & 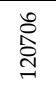 & $\begin{array}{l}\infty \\
\text { స్స } \\
\text { స్ }\end{array}$ \\
\hline ORF & & M & 尺 & $\infty$ & $\vec{\infty}$ & $\mathscr{\infty}$ & $\infty$ & $\infty$ & \multicolumn{2}{|c|}{ \& } & 2 & ஜू & ๙ & 홍 & & \multicolumn{2}{|c|}{$\exists$} & $\exists$ & $\stackrel{ㅁ}{=}$ & & & $\mathbb{I}$ & & & \multicolumn{3}{|c|}{ జ్తి } & $\widetilde{\widetilde{\Omega}}$ & & $\stackrel{\mathbb{9}}{\stackrel{9}{9}}$ & $\stackrel{\stackrel{N}{\rightrightarrows}}{\sim}$ & \multicolumn{2}{|c|}{ I } & $\exists$ \\
\hline CpGV-SA & C & $\mathrm{T}$ & $\mathrm{T}$ & $\mathrm{T}$ & $T$ & $\mathrm{~T}$ & $\mathrm{~A}$ & A & $\mathrm{C}$ & $\mathrm{T}$ & A & $\mathrm{A}$ & A & $\mathrm{A}$ & $C$ & G & G & $\mathrm{A}$ & C & $\mathrm{A}$ & G & A & $\mathrm{T}$ & $T$ & $\mathrm{~T}$ & $\mathrm{~A}$ & $\mathrm{~A}$ & $\mathrm{~T}$ & $C$ & A & $C$ & A & $\mathrm{A}$ & $\mathrm{C}$ \\
\hline CpGV & $\mathrm{T}$ & C & G & C & C & $\mathrm{Ca}^{\mathrm{a}}$ & $C$ & G & $\mathrm{T}^{\mathrm{b}}$ & C & G & G & G & G & $\mathrm{T}$ & $\mathrm{C}$ & $\mathrm{A}$ & $\mathrm{T}$ & $\mathrm{G}^{\mathrm{c}}$ & G & $\mathrm{A}$ & G & $\mathrm{A}$ & $C$ & C & G & G & $C$ & $\mathrm{~T}$ & G & $\mathrm{T}$ & $\mathrm{C}^{\mathrm{d}}$ & C & $\mathrm{T}$ \\
\hline $\begin{array}{c}\text { Substitution } \\
\text { SA } \rightarrow \text { Ref }\end{array}$ & & & $\begin{array}{l}\text { مि} \\
\hat{1} \\
\dot{1}\end{array}$ & & & & $\stackrel{\wedge}{\stackrel{1}{i}}$ & & & & $\begin{array}{l}n \\
\hat{z} \\
z\end{array}$ & & & $\stackrel{\hat{\imath}}{i}$ & & & & 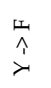 & & $\begin{array}{l}\overleftrightarrow{\hat{r}} \\
\hat{H}\end{array}$ & & & $\overrightarrow{\hat{i}}$ & & & & $\begin{array}{l}\hat{\imath} \\
\hat{y}\end{array}$ & $\begin{array}{l}\infty \\
\hat{j}\end{array}$ & & $\begin{array}{l}\stackrel{4}{\hat{j}} \\
\dot{>}\end{array}$ & $\stackrel{\overrightarrow{\hat{u}}}{\mathrm{u}}$ & $\overrightarrow{\hat{i}}$ & $\stackrel{\overrightarrow{\hat{x}}}{\dot{\varkappa}}$ & $\stackrel{+}{\hat{\&}}$ \\
\hline
\end{tabular}


The comparison identified a total of 821 SNPs between CpGV-SA and CpGV-E2, -I07, -I12, -M, and $-S$, of which 67 were novel (Table 1). The SNPs resulted in a total of 352 amino acid substitutions amongst the various CpGV isolates, of which 24 were unique to CPGV-SA. Two polymorphisms were detected at position 98024 (97937 in CpGV-M shown in Table 1) with CPGV-SA and -E2 both differing from the other isolates.

\subsection{Genotypic Variation within the CpGV-SA Isolate}

Intra specific variation was analysed by reassembling and mapping of the Illumina reads to the complete CpGV-SA genome sequence described above. For this analysis, a mean coverage of 2210.7 ( \pm 911.4 ) per nucleotide was achieved with a minimum of 152 and maximum of 4438 . A total of 66 genotypic SNVs were detected in the CpGV-SA read data when compared against the complete genome sequence (Table 2). The average quality was greater than Q30 for $95 \%$ for all reads utilized in detection of these SNVs. Of the $66 \mathrm{SNVs}, 22$ resulted in amino acid changes in protein sequences (Table 2). 
Table 2. Alignment of 66 genotypic SNVs identified within the CpGV-SA complete genome sequence. Variants which overlap with SNPs identified between the CpGV isolates are in light grey while those which overlap with SNPs unique to CpGV-SA (Table 1) are in dark grey. The corresponding ORF numbers are provided above the alignment for SNVs occurring within coding regions while any resulting amino acid substitutions are shown below. Nucleotides: $\mathrm{A}=\mathrm{Adenine}, \mathrm{C}=\mathrm{Cytosine}, \mathrm{G}=$ Guanine, $\mathrm{T}=$ Thymine and amino acids: $\mathrm{A}=$ Alanine, $\mathrm{R}=$ Arginine, $\mathrm{N}=$ Asparagine, $\mathrm{D}=$ Aspartic acid, $\mathrm{E}=\mathrm{Glutamic}$ acid $, \mathrm{G}=\mathrm{Glycine}, \mathrm{H}=\mathrm{Histidine}, \mathrm{I}=\mathrm{Isoleucine}$, $\mathrm{K}=$ Lysine, $\mathrm{M}=$ Methionine, $\mathrm{T}=$ Threonine, $\mathrm{Y}=$ Tyrosine, and $\mathrm{V}=$ Valine.

\begin{tabular}{|c|c|c|c|c|c|c|c|c|c|c|c|c|c|c|c|c|c|c|c|c|c|c|c|c|c|c|c|c|c|c|c|c|c|}
\hline CpGV-SA Position & ஜ్రి & జ్ & क્ర్రి & స్తి & 공 & 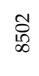 & 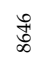 & ڤ్ & สี & $\begin{array}{l}\stackrel{2}{a} \\
\underset{\exists}{J}\end{array}$ & $\begin{array}{l}\text { 总 } \\
\text { 总 }\end{array}$ & 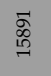 & $\stackrel{\text { }}{\Xi}$ & $\begin{array}{l}\text { N } \\
\text { No }\end{array}$ & 롬 & क्ञेत & 胥 & 苶 & స్ & 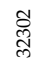 & 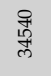 & 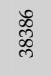 & $\begin{array}{l}\text { 今े } \\
\text { ò }\end{array}$ & 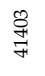 & 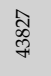 & 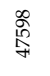 & $\begin{array}{l}\text { जo } \\
\text { b }\end{array}$ & $\begin{array}{l}\text { 㟥 } \\
\text { के }\end{array}$ & $\frac{8}{\vec{t}}$ & 旁 & $\begin{array}{l}\text { Iี } \\
\text { iี }\end{array}$ & $\begin{array}{l}\text { 월 } \\
\text { in }\end{array}$ & 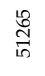 \\
\hline ORFs & \multicolumn{5}{|c|}{10} & \multicolumn{2}{|c|}{11} & & 12 & \multicolumn{2}{|c|}{15} & 20 & \multicolumn{2}{|c|}{22} & & & & & & 37 & 41 & 46 & 48 & \multicolumn{2}{|c|}{$\begin{array}{l}50 / 51 \\
52 \mathrm{~b}\end{array}$} & 5 & & 60 & \multicolumn{2}{|c|}{61} & & & \\
\hline CpGV-SA & $\mathrm{T}$ & $\mathrm{A}$ & $\mathrm{A}$ & $\mathrm{T}$ & G & $\mathrm{C}$ & G & A & G & $\mathrm{C}$ & $\mathrm{T}$ & $\mathrm{T}$ & $\mathrm{C}$ & A & A & $\mathrm{A}$ & A & $\mathrm{C}$ & A & G & A & A & A & G & $\mathrm{T}$ & $\mathrm{C}$ & $\mathrm{C}$ & G & $\mathrm{T}$ & $\mathrm{C}$ & G & $\mathrm{T}$ & A \\
\hline Variant & C & G & $\mathrm{G}$ & C & $\mathrm{A}$ & $\mathrm{T}$ & $\mathrm{A}$ & $\mathrm{T}$ & $\mathrm{A}$ & $\mathrm{T}$ & $\mathrm{A}$ & C & $\mathrm{T}$ & G & $\mathrm{T}$ & $\mathrm{T}$ & $\mathrm{T}$ & $\mathrm{A}$ & G & $\mathrm{T}$ & G & $\mathrm{T}$ & G & $\mathrm{A}$ & C & $\mathrm{T}$ & $\mathrm{T}$ & $\mathrm{A}$ & $\mathrm{A}$ & G & $\mathrm{T}$ & C & C \\
\hline Variant Coverage & ๕ू & 胥 & 总 & $\underset{\mathbb{I}}{\stackrel{D}{2}}$ & कै & $\underset{\substack{\infty \\
\sim}}{\sim}$ & $\stackrel{\mathbb{\Xi}}{\mathrm{E}}$ & $\stackrel{\widetilde{d}}{\widetilde{్}}$ & 赵 & 总 & 孛 & 苛 & $\stackrel{\infty}{\stackrel{2}{N}}$ & 茨 & h్ & $\stackrel{\mathscr{2}}{\text { สิ }}$ & สี & 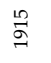 & $\widetilde{\sigma}$ & $\tilde{\text { in }}$ & $\stackrel{\infty}{n}$ & $\overrightarrow{0}$ & $\frac{\widehat{D}}{\infty}$ & 焘 & 胥 & $\begin{array}{c}\infty \\
\stackrel{3}{6}\end{array}$ & $\begin{array}{l}\infty \\
\substack{\infty \\
\rightarrow \rightarrow}\end{array}$ & 片 & $\begin{array}{l}\text { ते } \\
\text { ते }\end{array}$ & $\underset{\substack{\infty \\
\stackrel{\infty}{N}}}{ }$ & 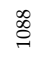 & I & $\stackrel{\mathbb{J}}{\mathbb{J}}$ \\
\hline Amino Acid Change & & & $\hat{i}$ & & $\begin{array}{l}\overrightarrow{\hat{x}} \\
\dot{4}\end{array}$ & & & & $\hat{\hat{\leftrightarrow}}$ & $\hat{\hat{\psi}}$ & $\begin{array}{l}\lambda \\
\hat{z}\end{array}$ & $\begin{array}{l}\mathbb{\hat { H }} \\
\stackrel{H}{2}\end{array}$ & & $\begin{array}{l}\stackrel{\mathbb{1}}{\hat{r}} \\
\stackrel{1}{2}\end{array}$ & & & & & & & & $\underline{z_{1}}$ & $\begin{array}{l}\text { U } \\
\hat{\rho}\end{array}$ & & & $\hat{\hat{\psi}}$ & $\hat{\hat{i}}$ & & $\overrightarrow{\hat{z}}$ & $\hat{\hat{\varkappa}}$ & & & \\
\hline
\end{tabular}

\begin{tabular}{|c|c|c|c|c|c|c|c|c|c|c|c|c|c|c|c|c|c|c|c|c|c|c|c|c|c|c|c|c|c|c|c|c|c|}
\hline CpGV-SA Position & $\begin{array}{l}\text { 今̂̀ } \\
\text { in }\end{array}$ & $\begin{array}{l}\text { ते } \\
\text { है }\end{array}$ & $\begin{array}{l}\text { 芯 } \\
\text { तै }\end{array}$ & $\begin{array}{l}\text { ồ } \\
\text { in }\end{array}$ & $\begin{array}{l}\text { के } \\
\text { îे }\end{array}$ & $\begin{array}{l}\text { 范 } \\
\text { ते }\end{array}$ & 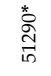 & $\begin{array}{l}\text { 总 } \\
\text { in }\end{array}$ & $\begin{array}{l}\overrightarrow{0} \\
\text { in } \\
\text { in }\end{array}$ & $\begin{array}{l}\text { o } \\
\text { in }\end{array}$ & $\begin{array}{l}\text { ⿵人 } \\
\text { in }\end{array}$ & $\begin{array}{l}\text { oొ } \\
\text { in }\end{array}$ & $\frac{f}{i t}$ & $\frac{\text { 焉 }}{\text { in }}$ & $\begin{array}{l}\text { 恣 } \\
\text { in }\end{array}$ & 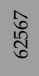 & $\begin{array}{l}\overrightarrow{8} \\
\text { 古 }\end{array}$ & 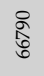 & స్ & $\begin{array}{l}\text { 苦 } \\
\text { d. }\end{array}$ & 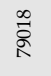 & 恋 & $\begin{array}{l}\text { 䓌 } \\
\text { 密 }\end{array}$ & $\begin{array}{l}\text { ष̃ } \\
\text { ळू }\end{array}$ & $\begin{array}{l}\text { 心ే } \\
\text { ळू }\end{array}$ & 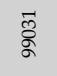 & $\begin{array}{l}\text { Nั" } \\
\text { ڤ్ }\end{array}$ & $\stackrel{\infty}{\stackrel{\infty}{\Xi}}$ & $\begin{array}{l}\text { 号 } \\
\text { 恶 }\end{array}$ & $\begin{array}{l}\stackrel{0}{N} \\
=\end{array}$ & 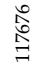 & $\begin{array}{l}\bar{\delta} \\
\stackrel{\circ}{\Xi} \\
\exists\end{array}$ & $\begin{array}{l}\text { के } \\
\text { त्रे }\end{array}$ \\
\hline ORFs & & & & & & & & & & & & & & & & 77 & $82 \mathrm{~b}$ & \multicolumn{2}{|c|}{84} & 89 & 96 & 99 & 110 & & 115 & 116 & 117 & 128 & 131 & \multicolumn{2}{|c|}{135} & 139 & 141 \\
\hline CpGV-SA & C & $\mathrm{T}$ & $\mathrm{A}$ & $\mathrm{T}$ & $\mathrm{C}$ & $\mathrm{T}$ & $\mathrm{T}$ & $\mathrm{G}$ & $\mathrm{T}$ & $\mathrm{T}$ & $\mathrm{T}$ & $\mathrm{T}$ & C & $\mathrm{C}$ & $\mathrm{T}$ & $\mathrm{T}$ & $\mathrm{T}$ & $\mathrm{T}$ & $\mathrm{C}$ & $\mathrm{A}$ & $\mathrm{A}$ & G & $\mathrm{G}$ & $\mathrm{C}$ & $\mathrm{C}$ & $\mathrm{A}$ & $\mathrm{A}$ & $\mathrm{T}$ & $\mathrm{T}$ & $\mathrm{T}$ & $\mathrm{C}$ & $\mathrm{G}$ & $\mathrm{C}$ \\
\hline Variant & $\mathrm{T}$ & $\mathrm{G}$ & $\mathrm{T}$ & G & $\mathrm{A}$ & $\mathbf{A}$ & $\mathrm{C}$ & C & $\mathrm{A}$ & C & $\mathrm{A}$ & $\mathrm{A}$ & $\mathrm{T}$ & $\mathrm{T}$ & $\mathrm{A}$ & $\mathrm{G}$ & C & C & $\mathrm{T}$ & G & G & $\mathrm{A}$ & $\mathrm{A}$ & $\mathrm{T}$ & G & $G$ & $G$ & C & C & C & $\mathrm{T}$ & $\mathrm{A}$ & $\mathrm{T}$ \\
\hline Variant Coverage & 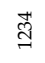 & 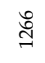 & 웜 & 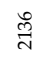 & $\frac{R}{m}$ & 용 & ন্ & 娄 & 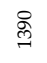 & 孪 & 蛋 & 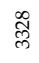 & సี & 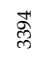 & ह్ & & Iี & $\infty$ & तु & $\stackrel{\mathbb{N}}{\mathbb{N}}$ & $\stackrel{\infty}{\infty}$ & 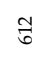 & $\stackrel{\mathbb{N}}{\triangle}$ & ू̆ & 嚕 & 吕 & $\underset{\mathbb{Z}}{\mathbb{Z}}$ & 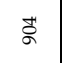 & 曹 & $\stackrel{\circ}{\stackrel{ }{ㄱ}}$ & $\stackrel{8}{\circ}$ & g్ & 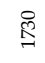 \\
\hline Amino Acid Change & & & & & & & & & & & & & & & & $\begin{array}{l}\text { 令 } \\
\text { in }\end{array}$ & & & & & & $\overrightarrow{\hat{i}}$ & & & 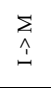 & $\begin{array}{l}\overleftarrow{\hat{i}} \\
\hat{H}\end{array}$ & 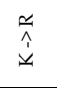 & & 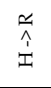 & $\begin{array}{l}\dot{\hat{b}} \\
\hat{i}\end{array}$ & & $\overrightarrow{\hat{i}}$ & $\stackrel{\overrightarrow{\hat{\epsilon}}}{\mathrm{s}}$ \\
\hline
\end{tabular}


Out of all 821 SNPs detected between the CpGV-SA and CpGV-M, I12, E2, S and I07 isolates, 31 were potentially due to genotypic variation within the South African isolate (highlighted light grey in Table 2). Of the 31 SNVs, 11 were identified to overlap with SNPs unique to CpGV-SA (highlighted dark grey in Table 2). For each of these, the genotypic variant identified matched the respective nucleotide present in the other CpGV isolates. The resulting number of unique SNPs present within the CpGV-SA isolate could therefore range between 56 and 67 depending on the genotypic mixture. The remaining 36 SNVs detected in CpGV-SA were not present in the SNP data. A single nucleotide, at position 51290 in the CPGV-SA genome, was detected to have three variants, either a $\mathrm{T}$ (as in the CpGV-SA reference sequence), an A or a C (marked * in Table 2).

Analysis of INDELs in CPGV-SA was also performed to identify variation within this and the genomes of the other CPGV isolates. Of the INDELs mentioned above, those occurring in ORF cp31, cp37 and cp62 of the CPGV-SA isolate, were absent amongst some of the genotypic variants resulting in the preservation of ORF as annotated in the other CpGV isolates. For example, CpGV-SA ORF cp62 was truncated due to the insertion of a single nucleotide at position 51479. However, this nucleotide was revealed to have considerable variation amongst the CpGV-SA Illumina data, with numerous reads indicating the absence of this insertion, resulting in an ORF like that of cp62 in the CpGV-S and E2 isolates. Similarly, other INDELs identified in CpGV-SA were found to match some which had been previously identified in other CpGV isolates resulting in similar ORFs. An example of this was CPGV-SA cp6, whereby an INDEL present within genotypic variants was identical to an ORF previously detected only in CpGV-S cp6.

\subsection{Phylogenetic Analysis}

The phylogeny of the six CpGV isolates was determined by alignment and analysis of the complete genome sequences (Figure 1).

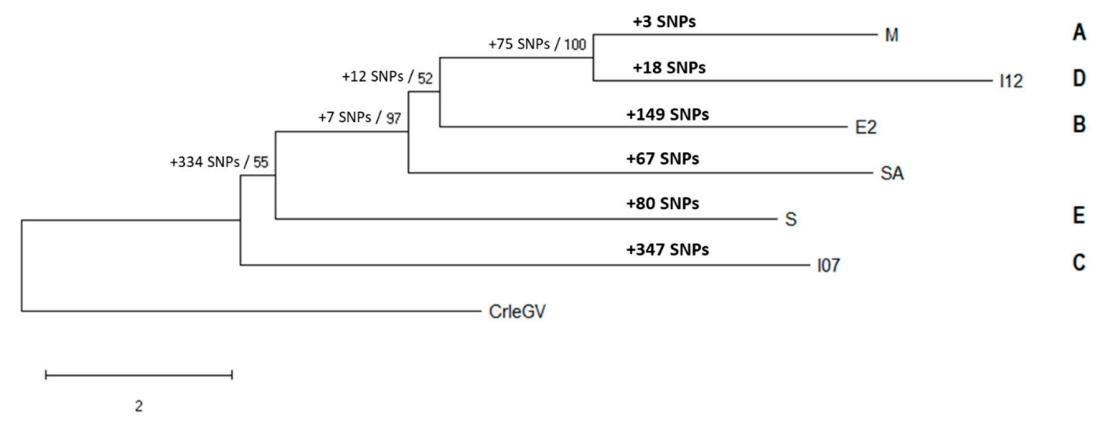

Figure 1. CpGV phylogeny based on complete genome sequences for isolates from groups A-E and CpGV-SA. The number of unique SNPs present in each isolate is shown above the branches in bold. The number of SNPs per grouping is shown at each node alongside the bootstrap values. CpGV groups are indicated to right of the respective isolate.

Phylogenetic analysis of the CPGV isolates placed the South African isolate basal to isolates M, I12 and E2 and distal to $S$ and $I 07$ with strong bootstrap support calculated (97\%). Although low bootstrap support was calculated for the CpGV-E2, I07 and S isolates, all branches generated were dichotomous. CpGV-SA was also identified to have more unique SNPs (67) than the CPGV-M and I12 isolates (3 and 18 , respectively).

\section{Discussion}

Initial characterization of CPGV-SA using restriction enzyme analysis of genomic DNA provided the first evidence that CPGV-SA is not only genetically different from other sequenced CpGV isolates, but also of mixed genotypes [27]. This observation prompted further in-depth genetic characterization by sequencing of the complete genome using the Illumina MiSeq platform, achieving an average coverage of $545.4 \pm 191.3$, considerably higher than that attained for the CpGV-M, I12, E2, S and I07 
isolates [7]. This platform can generate large quantities of data with extremely low error rates of approximately $0.24 \%$ per base pair [31]. This not only enabled the comparative analysis of intra-species SNPs between CpGV-SA and other sequenced CpGV isolates, but also evaluation of genotypic variation or SNVs within the isolate.

Comparison of the CpGV-SA genome with other sequenced genomes identified a total of 821 SNPs compared to 788 reported previously by [7]. However, a comparison of the data generated in this study shows that only 526 of the 788 SNPs are still detectable. This difference is likely due to changes introduced during the alignment of all six genome sequences, with the inclusion of the South African isolate changing penalties incurred during gap openings and the degree to which these are extended. Therefore, instead of a direct comparison of the number of SNPs as previously reported, changes in SNP patterns, particularly those in ORFs, were evaluated. The analysis revealed that of the 664 SNPs identified between all six isolates, 67 were unique to CpGV-SA and the majority of these resulted in amino acid changes in protein sequences. Interestingly, phylogenetic analysis based on alignment of six CpGV complete genomes placed the CpGV-SA isolate basal to CpGV-M, I12 and E2 with no definite classification into any of the previously defined genogroups $[5,6]$. When combining the result of this tree with the SNP analysis and REN profiles [27], it appears that CpGV-SA is a genetic variant of previously described isolates and likely representative of a new phylogenetic group.

Analysis of intra species variation by reassembly and mapping of Illumina reads to the complete CpGV-SA genome sequence showed that, similar to previously analyzed CpGV isolates [5], the wild-type CpGV-SA isolate consists of a mixture of genotypes. Such a mixture of genotypes, as is found in natural populations of most wild-type baculovirus isolates [32,33], has important implications for the control of CM in South Africa where commercial formulations based on the Mexican isolate are extensively applied, risking the development of resistance in field CM populations as was the case in Europe. Notably, CpGV-SA has type I resistance breaking potential characteristic of other isolates currently registered in Europe and North America as biological control agents. Further studies are required to test the ability of CpGV-SA to overcome other types of resistance which, to date, is only achieved by the CpGV-E2 isolate [21,34].

In summary, the results of this study indicate that CpGV-SA is a unique isolate which is genetically distinct from those previously described and probably representative of a new genogroup. Although not exhibiting superior biological activity when compared to CpGV-M [26,27], the discovery of CpGV-SA may provide an additional valuable tool to enable alternating application of products based on diverse isolates for resistance management in South Africa and possibly in Europe where such resistance already occurs.

Author Contributions: C.M.K., M.P.H. and S.D.M. supervised the project; C.M.K., M.P.H. and S.D.M. and B.M. conceived and designed the experiments; B.M. performed the experiments; B.M. and M.D.J. analysed the data; B.M., C.M.K. and M.D.J. wrote the paper, and all authors thoroughly revised it.

Funding: This work is based on the research supported in part by the National Research Foundation of South Africa: South African Research Chairs Initiative (SARChi) Grant number: UID119609, the National Research Foundation of South Africa, and the Rhodes University Research Council.

Acknowledgments: The authors would like to thank Craig Chambers and River Bioscience (Pty) Ltd. for providing viral samples.

Conflicts of Interest: The authors declare no conflict of interest. The sponsors had no role in the design, execution, interpretation, or writing of the study.

\section{References}

1. Lacey, L.A.; Thomson, D.; Vincent, C.; Arthurs, S.P. Codling moth granulovirus: A comprehensive review. Biocontrol. Sci. Technol. 2008, 18, 639-663. [CrossRef]

2. Blomefield, T.L.; Giliomee, J.H. Validation of the Phenology Model for the Codling Moth, Cydia pomonella (Lepidoptera: Tortricidae), in South African Pome Fruit Orchards. African Entomol. 2014, 22, 30-48. [CrossRef] 
3. Rezapanah, M.; Shojai-Estabragh, S.; Huber, J.; Jehle, J.A. Molecular and biological characterization of new isolates of Cydia pomonella granulovirus from Iran. J. Pest Sci. 2008, 81, 187-191. [CrossRef]

4. Tanada, Y. Granulosis virus of codling moth Carpocapsa pomonella (linnaeus) Olethreutidae lepidoptera). J. Insect Pathol. 1964, 6, 378.

5. Eberle, K.E.; Sayed, S.; Rezapanah, M.; Shojai-Estabragh, S.; Jehle, J.A. Diversity and evolution of the Cydia pomonella granulovirus. J. Gen. Virol. 2009, 90, 662-671. [CrossRef] [PubMed]

6. Gebhardt, M.M.; Eberle, K.E.; Radtke, P.; Jehle, J.A. Baculovirus resistance in codling moth is virus isolate-dependent and the consequence of a mutation in viral gene pe38. Proc. Natl. Acad. Sci. 2014, 111, 15711-15716. [CrossRef] [PubMed]

7. Wennmann, J.T.; Radtke, P.; Eberle, K.E.; Alletti, G.G.; Jehle, J.A. Deciphering single nucleotide polymorphisms and evolutionary trends in isolates of the Cydia pomonella granulovirus. Viruses 2017, 9, 227. [CrossRef] [PubMed]

8. Huber, J.; Hunter-Fujita, F.R.. Insect Viruses and Pest Management; Wiley: Hoboken, NJ, USA, 1998; Volume 491, pp. 201-215.

9. Sauphanor, B.; Berling, M.; Toubon, J.; Reyes, M.; Delnatte, J.; Allemoz, P. Carpocapse des pommes. Cas de resistance au virus de la granulose en vergers biologique. Phytoma La Def. des Veg. 2006, 590, 24-27.

10. Fritsch, E.; Undorf-spahn, K.; Kienzle, J.; Huber, J. Apfelwickler-Granulovirus: Erste Hinweise auf Unterschiede in der Empfindlichkeit lokaler Apfelwickler-populationen. Nachrichtenbl. Deut. Pflanzenschutzd 2005, 57, 29-34.

11. Asser-Kaiser, S.; Fritsch, E.; Undorf-Spahn, K.; Kienzle, J.; Eberle, K.E.; Gund, N.A.; Reineke, A.; Zebitz, C.P.W.; Heckel, D.G.; Huber, J.; et al. Rapid emergence of baculovirus resistance in codling moth due to dominant, sex-linked inheritance. Science 2007, 317, 1916-1918. [CrossRef] [PubMed]

12. Eberle, K.E.; Jehle, J.A. Field resistance of codling moth against Cydia pomonella granulovirus (CpGV) is autosomal and incompletely dominant inherited. J. Invertebr. Pathol. 2006, 93, 201-206. [CrossRef]

13. Jehle, J.A.; Schulze-Bopp, S.; Undorf-Spahn, K.; Fritsch, E. Evidence for a second type of resistance against Cydia pomonella granulovirus in field populations of codling moths. Appl. Environ. Microbiol. 2017, 83, 1-13. [CrossRef] [PubMed]

14. Eberle, K.E.; Asser-Kaiser, S.; Sayed, S.M.; Nguyen, H.T.; Jehle, J.A. Overcoming the resistance of codling moth against conventional Cydia pomonella granulovirus (CPGV-M) by a new isolate CpGV-I12. J. Invertebr. Pathol. 2008, 98, 293-298. [CrossRef] [PubMed]

15. Berling, M.; Rey, J.B.; Ondet, S.J.; Tallot, Y.; Soubabère, O.; Bonhomme, A.; Sauphanor, B.; Lopez-Ferber, M. Field trials of CpGV virus isolates overcoming resistance to CpGV-M. Virologica Sinca 2009, 24, 470-477. [CrossRef]

16. Berling, M.; Blachere-Lopez, C.; Soubabere, O.; Lery, X.; Bonhomme, A.; Sauphanor, B.; Lopez-Ferber, M. Cydia pomonella granulovirus genotypes overcome virus resistance in the codling moth and improve virus efficiency by selection against resistant hosts. Appl. Environ. Microbiol. 2009, 75, 925-930. [CrossRef]

17. Zingg, D. Madex Plus and Madex I12 overcome Virus Resistance of Codling Moth. In Proceedings of the Ecofruit-13th International Conference on Cultivation Technique and Phytopathological Problems in Organic Fruit-Growing, Weinsberg, Germany, 18-20 February 2008; pp. 256-260.

18. Zichová, T.; Falta, V.; Kocourek, F.; Stará, J. Differences in the susceptibility of codling moth populations to Cydia pomonella granulovirus in the Czech Republic. Hortic. Sci. 2011, 38, 21-26. [CrossRef]

19. Schmitt, A.; Bisutti, I.L.; Ladurner, E.; Benuzzi, M.; Sauphanor, B.; Kienzle, J.; Zingg, D.; Undorf-Spahn, K.; Fritsch, E.; Huber, J.; et al. The occurrence and distribution of resistance of codling moth to Cydia pomonella granulovirus in Europe. J. Appl. Entomol. 2013, 137, 641-649. [CrossRef]

20. Kienzle, J.; Zimmer, J.; Volk, F.; Zebitz, C.P.W. Field tests with Madex Plus against CpGV-resistant codling moth populations in organic orchards in 2006. In Proceedings of the Ecofruit-13th International Conference on Cultivation Technique and Phytopathological Problems in Organic Fruit-Growing, Weinsberg, Germany, 18-20 February 2008; pp. 261-264.

21. Sauer, A.J.; Schulze-Bopp, S.; Fritsch, E.; Undorf-Spahn, K.; Jehle, J.A. A third type of resistance to Cydia pomonella granulovirus in codling moths shows a mixed Z-linked and autosomal inheritance pattern. Appl. Environ. Microbiol. 2017, 83. [CrossRef]

22. Hortgro Key Deciduous Fruit Statistics. 2017. Available online: https://www.hortgro.co.za/markets/keydeciduous-fruit-statistics/ (accessed on 27 June 2019). 
23. Lounsbury, C.P. The Codling Moth; Townshend, Taylor and Snashall, Printers: Cape Town, South Africa, 1898; pp. 1-8.

24. Charleston, D.; Kfir, R.; Rensburg, N.; van Barnes, B.; Hatting, V.; Conlong, D.E. Integrated pest management in South Africa. In Integrated Pest Management in the Global Arena; Karim, M., Maredia, D., Dakouo, D., Mota-Sanchez, Eds.; CABI publishing: Wallingford, UK, 2009; pp. 169-196. ISBN 0851996523.

25. Hatting, J.L.; Moore, S.D.; Malan, A.P. Microbial control of phytophagous invertebrate pests in South Africa: Current status and future prospects. J. Invertebr. Pathol. 2018, 1-13. [CrossRef]

26. Chambers, C.B. Production of Cydia pomonella granulovirus (CpGV) in a Heteralogous Host, Thaumatotibia leucotreta (Meyrick) (False Codling Moth). Ph.D. Thesis, Rhodes University, Grahamstown, South Africa, 2015.

27. Motsoeneng, B.M. Genetic and Biological Characterisation of a Novel South African Cydia pomonella Granulovirus (CpGV-SA) Isolate. Master Thesis, Rhodes University, Grahamstown, South Africa, 2016.

28. Luque, T.; Finch, R.; Crook, N.; O'Reilly, D.R.; Winstanley, D. The complete sequence of the Cydia pomonella granulovirus genome. J. Gen. Virol. 2001, 82, 2531-2547. [CrossRef]

29. Kumar, S.; Stecher, G.; Li, M.; Knyaz, C.; Tamura, K. MEGA X: Molecular evolutionary genetics analysis across computing platforms. Mol. Biol. Evol. 2018, 35, 1547-1549. [CrossRef] [PubMed]

30. Lange, M.; Jehle, J.A. The genome of the Cryptophlebia leucotreta granulovirus. Virology 2003, 317, $220-236$. [CrossRef]

31. Pfeiffer, F.; Gröber, C.; Blank, M.; Händler, K.; Beyer, M.; Schultze, J.L.; Mayer, G. Systematic evaluation of error rates and causes in short samples in next-generation sequencing. Sci. Rep. 2018, 8, 1-14. [CrossRef] [PubMed]

32. Cory, J.S.; Myers, J.H. The Ecology and Evolution of Insect Baculoviruses. Annu. Rev. Ecol. Evol. Syst. 2003, 34, 239-272. [CrossRef]

33. Erlandson, M.A. Genetic variation in field populations of baculoviruses: Mechanisms for generating variation and its potential role in baculovirus epizootiology. Virol. Sin. 2009, 24, 458-469. [CrossRef]

34. Alletti, G.G.; Sauer, A.J.; Weihrauch, B.; Fritsch, E.; Undorf-Spahn, K.; Wennmann, J.T.; Jehle, J.A. Using next generation sequencing to identify and quantify the genetic composition of resistance- breaking commercial isolates of Cydia pomonella granulovirus. Viruses 2017, 9, 250. [CrossRef]

(C) 2019 by the authors. Licensee MDPI, Basel, Switzerland. This article is an open access article distributed under the terms and conditions of the Creative Commons Attribution (CC BY) license (http://creativecommons.org/licenses/by/4.0/). 